Forum 2017 · 32:156

DOI 10.1007/s12312-017-0233-y

Online publiziert: 7. März 2017

○) Springer Medizin Verlag GmbH 2017

V. Grünwald • T. Steiner für die interdisziplinäre Arbeitsgruppe Nierentumore
der DKG

\title{
Stellungnahme zur adjuvanten Therapie des fortgeschrittenen Nierenzellkarzinoms
}

Unterschiede der beiden Studien be-

Tyrosinkinaseinhibitoren (TKI) sind ein Standard zur Behandlung des metastasierten Nierenzellkarzinoms (NCC). Aktuelle Studien untersuchen nun den Einsatz von verschiedenen zielgerichteten Substanzen in der adjuvanten Situation. Die ASSURE (Adjuvant Sorafenib or Sunitinib for Unfavorable REnal carcinoma) Studie untersuchte den adjuvanten Einsatz von Sorafenib oder Sunitinib über 1 Jahr im Vergleich zu Placebo bei Patienten mit fortgeschrittenem NCC und konnte 1943 Patienten einschließen [1]. Unter den volldosierten TKI war die Abbruchrate über $40 \%$, was zu einer Reduktion der TKIDosis nach Einschluss von 1323 Patienten führte und die Verträglichkeit der Therapie steigerte. Insgesamt konnte die Studie keine Verbesserung des Erkrankungsfreien Überlebens (DFS) zugunsten der TKI Behandlung zeigen (Sunitinib: 5,8 J.; Sorafenib: 6,1 J.; Placebo: 6,6 J.)[1]. Unter der Behandlung sind 5 therapiebedingte Todesfälle aufgetreten.

Die S-TRAC (Sunitinib Trial in Adjuvant Renal Carcinoma) Studie untersuchte die Einnahme von Sunitinib oder Placebo über 1 Jahr an 615 Teilnehmern. Der primäre Endpunkt der Studie wurde erreicht (DFS: 6,8 vs. 5,6 J.; HR 0,761, $p=0,03$ ), allerdings liegt das Gesamtüberleben nur als Zwischenanalyse vor [2]. Nach 5 Jahren beträgt die DFS-Rate 59,3\% (Sunitinib) bzw. 51,3\% (Placebo). Es wurden keine behandlungsassoziierten Todesfälle berichtet. 54,2\% konnten die volle Dosis für Sunitinib erhalten. stehen zum einen in der Größe (1943 vs. 615 Patienten), zum anderen in der Patientenselektion. Während S-TRAC lediglich klarzellige Nierenzellkarzinome eingeschlossen hat, wurden in ASSURE auch Patienten mit nicht-klarzelligem NCC (21\%), einschließlich sarkomatoide NCC, eingeschlossen. Darüber hinaus wurden $9 \%$ der Patienten im Stadium I in ASSURE aufgenommen. Allerdings zeigte die Analyse der Subgruppen in ASSURE den gleichen Trend der Gesamtstudie. Sowohl für Tumorgröße (T1-2 vs. T3-4), Stadium (AJCC I-II vs. III-IV), Histologie (klarzellig vs. andere) als auch für das Grading (G1-2, G3, G4) konnte kein Vorteil durch eine TKI Behandlung gezeigt werden [1].

Der hohe Anteil an Grad 3-4 Toxizitäten war in beiden Studien vergleichbar. In S-TRAC wurde eine Zunahme der schweren Toxizitäten unter Sunitinib (62 vs. $21 \%$ ) beobachtet. In ASSURE fanden sich unter Sunitinib $63 \%$, Sorafenib $72 \%$ und unter Placebo $25 \%$ schwere unerwünschte Ereignisse ( $\geq$ Grad 3).

Zusammenfassend konnte mit S-TRAC erstmalig eine Verbesserung des DFS für Sunitinib gezeigt werden, allerdings war die Behandlung mit einer erheblichen Toxizität verbunden. Die weitaus größere ASSURE Studie war hingegen negativ. Die Grundlage der Bewertung stellt momentan der intermediäre Surrogatendpunkt DFS dar. Endgültige Überlebensdaten, die eine bessere
Einordnung in den Kontext ermöglichen, werden erst in mehreren Jahren erwartet. Trotz der Unterschiede der Patientenkollektive und des DFS-Vorteils der S-TRAC Studie ist damit aktuell die Wertigkeit einer adjuvanten Therapie unklar und kann damit nicht empfohlen werden. Eine adjuvante Therapie sollte daher weiter in klinischen Studien untersucht werden. Weitere Studien (ATLAS, EVEREST, PROTECT, SORCE) sind unterwegs und werden uns helfen, die zukünftige Rolle der zielgerichteten adjuvanten Therapie zu definieren.

\section{Korrespondenzadresse}

Prof. Dr. Viktor Grünwald

Medizinische Hochschule Hannover

Klinik für Hämatologie, Hämostaseologie,

Onkologie und Stammzelltransplantation

Carl-Neuberg-Str. 1

30625 Hannover

Tel: 05115323021

E-Mail: Gruenwald.Viktor@MH-Hannover.de

\section{Literatur}

1. Haas NB, Manola J, Uzzo RG et al (2016) Adjuvant sunitinib or sorafenib for high-risk, non-metastatic renal-cell carcinoma (ECOG-ACRIN E2805): a double-blind, placebo-controlled, randomised, phase 3 trial. Lancet 387:2008-2016. doi:10.1016/ S0140-6736(16)00559-6

2. Ravaud A, Motzer RJ, Pandha HS et al (2016) Adjuvant Sunitinib in High-Risk Renal-Cell Carcinoma after Nephrectomy. N Engl J Med. doi:10.1056/ NEJMoa1611406 